leaves, to rats and understanding of the relevant mechanisms governing the intestinal absorption and presystemic elimination. To define the ginkgo compounds to be studied, literature informatics-guided chemical profiling revealed that GBE50 extract contained 72 ginkgo constituents, including terpene lactones, flavonols, flavones, an isoflavone, biflavones, flavanols, and carboxylic acids, at levels ranging from 0.01 to $55.3 \mathrm{mg} / \mathrm{g}$. Among the ginkgo constituent groups were the terpene lactones and the flavonols that were significantly measurable in plasma after p.o. administration of GBE50 extract to rats. The intestinal absorption of terpene lactones appeared to be dictated by their intermediate membrane permeability, while the influences of MDR-1-and MRP-2-mediated intestinal efflux and the presystemic metabolism and biliary excretion might be relatively limited. Because of their deglycosylation absent in the small intestine and relatively slow presystemic elimination, many intact flavonol glycosides appeared in the rat plasma albeit with a limited extent of absorption. Colonic deglycosylation of the flavonol glycosides occurred and the glucuronides of flavonol aglycones were also measured in the plasma. Although some biflavones also had relatively high abundance in GBE50 extract, these ginkgo constituents were not measured in the rat plasma because of their poor solubility and poor permeability that hindered the intestinal absorption. The levels of the remaining ginkgo constituents in GBE50 extract were too low to be measured in the rat plasma. The current study enabled us to better understand the nature of systemic exposure to ginkgo compounds after p. o. administration of GBE50 extract and to more precisely implement multicomponent PK study of the extract.

\title{
BIOPHARMACEUTICAL ASPECTS OF THE DEVELOPMENT OF A PHYTOECDISTEROIDS (PE) OINTMENT COMPOSITION
}

\author{
(C) Molokhova E. ${ }^{1}$, Lipin D. ${ }^{1}$, Volodin V. ${ }^{2}$
}

\author{
${ }^{1}$ Perm State Pharmaceutical Academy, Russia \\ ${ }^{2}$ Institute of Biology, Komi Scientific Centre, Ural Branch, Russian Academy of Science, Syktyvkar, Russia
}

PE are analogues of insect steroid hormones occurring in plants. The establishment of reparative activity of PE (1) makes it relevant to develop compositions on their base. PE molecules have six or more hydroxyl groups and hydrophobic core and therefore the influence of the ointment composition nature on the bioavailability of PE was trialed. In these investigations preparation Serpisten (mixture of 20-hydroxyecdisone and 25S-inocosterone obtained from the plant Serratula coronata L.) has been used. For this purpose three types of compositions have been prepared at a concentration of Serpisten $0.02 \%(w / w)$ : hydrophobic (lanolin and vaseline - C1), hydrophilic (3\% sodium carboxymethyl cellulose gel - C2), emulsive (aerosil, vaseline oil, water purified, monoglyceride purified C3). Biopharmaceutical evaluation of prepared formulations has been performed using drug releasing from the compositions by dialysis through a membrane with subsequent determination of the release profile in the $U V$ region at $\lambda=240 \mathrm{~nm}$. The concentration of $P E$ was determined at 30, 60 and 120 minutes of dialysis. The test revealed that the PE release from the $\mathrm{C} 3$ was al- most entirely (100.2\%), while from C276.5\% of the total amount of PE was passed to aqueous medium, and from $\mathrm{C} 1$ only $37.5 \%$. During the rheological studies it has been established that the shear stress at the $\mathrm{C} 1$ was less than $80 \mathrm{~Pa}^{*} \mathrm{~s}$ at $\mathrm{C} 2-300 \mathrm{~Pa}^{*} \mathrm{~s}$ and at C3 $156 \mathrm{~Pa}^{\star} \mathrm{S}$. C3 is the closest to the «Bepanthen» ointment (Bayer, Germany), which has the wound-healing activity (211 $\mathrm{Pa}^{\star} \mathrm{s}$.). For further PE reparative properties trials, C3 have been chosen, for this test linear aseptic wound model has been used. In five days it was found that, the scar strength in experimental group was $281.6 \pm 40.4 \mathrm{gr}$., in control group $160.8 \pm 18.0 \mathrm{gr}$. and in intact $-123.4 \pm 20.0$ gr. This data are higher 1.75 times in experimental group, compared with a control group $(p=0.021)$ and 2.28 times in the intact $(p=0.009)$. The result of this work was the development of preparing technology topical emulsive formulation having the high bioavailability.

Reference: (1) Meybeck A, Bont F. 1990. Ecdysteroid-containing liposomes for wound healing and skin regeneration. Demande FR 2,637,182. (Chemical Abstracts 114: 30138r). 